\title{
Origine culturelle et réception théâtrale : étude du public de Trois de Mani Soleymanlou
}

\section{Ariane BELLEMARE}

Lorsqu'il est question de la norme et des marges, plusieurs éléments entrent en jeu. Toutefois, nous croyons que les questions liées à l'immigration et à la diversité culturelle représentent une porte d'entrée importante en ce qui a trait à ce rapport entre norme et marges. $\mathrm{Au}$ Québec, ce rapport est particulièrement trouble, comme tout le débat sur la Charte des valeurs québécoises 1'a montré. Tensions, conflits, crises : les mois qu'a duré ce débat auront laissé pour traces une société indécise quant à la place qu'elle doit laisser à l'Autre pour ne pas perdre sa propre identité. Bien que toutes les sphères de la société soient touchées par ce phénomène, les arts, et le théâtre en particulier, n'échappent pas à cette tendance, et la présence d'acteurs issus de diverses communautés culturelles se fait de plus en plus sentir. Alors, quand une pièce écrite par un immigrant et qui présente de surcroît plus d'une quarantaine d'acteurs issus de ce qu'il est courant de nommer la « diversité » est créée, nous ne pouvons la passer sous silence et nous pensons qu'il est important d'observer l'impact que cette pièce peut avoir dans la société pour tenter de comprendre si elle pourrait rassembler et non diviser les esprits quant aux questions liées à l'immigration.

C'est le cas avec Trois de Mani Soleymanlou, présentée en 2014 pour la première fois dans le cadre du Festival TransAmériques de Montréal. Soleymanlou est l'auteur de cette trilogie, mais également acteur et metteur en scène canadien d'origine iranienne ayant immigré en France puis au Canada dans sa jeunesse. L'œuvre comporte trois parties : en premier lieu, un monologue de Soleymanlou sur son rapport à l'identité et à l'immigration ; en deuxième lieu, un dialogue entre lui et Emmanuel Schwartz, acteur québécois francophone, et enfin, la partie contenant Trois, où plus d'une quarantaine d'acteurs issus de diverses communautés culturelles de Montréal échange sur des questions identitaires. Cette pièce donne lieu à un dialogue intraculturel où chacun dispose d'un espace pour communiquer. Le caractère intraculturel de la représentation est alors double. En effet, une première interaction a lieu entre le public et les artistes issus de divers groupes culturels. La seconde prend place sur scène grâce au dialogue interculturel des acteurs qui s'exprime librement. 
Cela fait quelque fois que nous utilisons le terme «intraculturel ». Contrairement à plusieurs théoriciens, nous l'avons préféré à celui d'interculturel pour plusieurs raisons. Développé par le théoricien Rustom Bharucha, le concept d'intraculturalité s'oppose d'entrée de jeu à une définition traditionnelle de l'interculturalité où l'accent est mis sur la division entre les deux cultures étudiées. D'après Ric Knowles, ce qui crée la principale différence entre les deux théories est que la notion d'intraculturalité renvoie à l'idée de « décrire la rencontre des cultures à l'intérieur même d'une nation plutôt qu'entre des nations » (32). De son côté, l'interculturalité se présente plutôt comme une hybridation volontaire des cultures, sur la rencontre intentionnelle entre des traditions théâtrales issues de différentes cultures. Or, l'intraculturalité privilégie plutôt l'échange culturel au sein d'une même société en tenant compte des particularités de chacun, et non à l'extérieur de la société par un observateur lointain. Trois répond donc à cette caractéristique de l'intraculturalité : elle a été créée dans le contexte de la nation québécoise avec divers acteurs issus de milieux culturels variés mais s'intégrant tous à cette nation. Ce faisant, nous avons choisi de préconiser le terme intraculturel à celui d'interculturel pour axer notre interprétation sur la société québécoise.

Dans cet article, nous présentons les résultats obtenus et tirés des recherches effectuées dans le cadre de notre mémoire de maîtrise intitulé La réception interculturelle de Trois de Mani Soleymanlou: étude de public. À partir de la trilogie de Soleymanlou, nous avons examiné comment les relations entre les individus issus de différentes communautés culturelles peuvent être amenées à changer au contact de l'art, et comment le théâtre amène à créer un lien entre les communautés culturelles pour les rapprocher. Après avoir établi les assises méthodologiques de l'étude, nous revenons en profondeur sur les résultats. Pour ce faire, nous mettons en évidence quelques-uns des phénomènes observés en lien avec les scènes qui ont marqué les spectateurs, la représentation plus ou moins fidèle de l'immigrant et la possibilité, pour Trois, d'influencer les spectateurs à changer leur perception de l'Autre à la suite de la représentation théâtrale. Notons ici que nous avons retenu seulement un bref échantillon des données recueillies pour ne garder que celles qui présentaient le plus de pertinence dans le rapport entre la norme et les marges.

Revenons tout d'abord sur la méthodologie de cette étude. L’intérêt de cette étude repose davantage sur la réaction du public par rapport à la pièce que le contenu de l'œuvre elle-même. En raison du caractère intraculturel de la pièce, l'hypothèse initiale se consacrait à la réception de la pièce, notamment la réaction des spectateurs ayant assisté aux représentations en fonction de 
leur origine culturelle. Nous nous sommes interrogée sur le public qui assistait aux représentations afin de savoir si de l'origine culturelle du spectateur émergeait une réception théâtrale différente. Nous supposons donc qu'en fonction de l'expérience du spectateur, en tant qu'immigrant (tel que le personnage principal) ou non-immigrant, la réception de l'œuvre est différente. Afin de valider cette hypothèse, nous avons réalisé des entretiens semi-dirigés avec un groupe de participants (quatre immigrants et six non-immigrants), le tout avec l'accord du comité d'éthique de l'Université du Québec à Trois-Rivières. Nous avions précédemment établi que les immigrants devaient être nés dans un autre pays et avoir immigré après l'âge de cinq ans pour qu'ils aient eu conscience du changement de pays. Le groupe des non-immigrants se composait quant à lui de spectateurs nés au Québec. Nous avons axé les questions des entretiens autour de trois grands thèmes, soit les questions liées à la pièce elle-même (scènes marquantes, moments forts), la représentation de l'immigrant (son côté réaliste ou fictif) et l'impact probable de la trilogie. Nous développons les résultats dans les paragraphes qui suivent.

Premièrement, notons que peu importe le groupe de participants, tous ont généralement apprécié la pièce. Les scènes ayant marqué le groupe composé d'immigrants se trouvent surtout dans la troisième partie de la pièce. Rappelons que cette partie présente une quarantaine d'acteurs dont des Iraniens, des Québécois, des Français, des Haïtiens, des Belges et toute une variété d'acteurs issus ou non de l'immigration. La scène de la bataille représente un moment marquant pour plusieurs spectateurs. C'est dans cette scène que culminent de vives discussions entre les personnages à propos des tensions culturelles au Québec. Bien que les spectateurs n'aient pas précisé en quoi cette scène les interpellait plus que les autres, nous croyons que c'est en raison de l'effet grandiose et spectaculaire provoqué par une mise en scène où, d'après les didascalies de l'auteur, « on se lance des flèches, ensuite on se tire dessus, on fait des grimaces, on s'arrache les cheveux » (Soleymanlou 145). En fait, la bataille est particulièrement poignante tant par son intensité physique que par son intensité émotionnelle : elle survient à la suite de vives discussions traitant de politique, de religion et de laïcité de l'État. Le spectateur peut donc être amené à revivre de façon figurée les débats ayant eu lieu au Québec en 2012 autour de la Charte des valeurs québécoises. Ces débats ont déchiré le Québec et laissé des marques vives chez plusieurs citoyens puisque la scène survient après des propos associés à cette période. Par ailleurs, la plupart des spectateurs ont noté que, lors de la discussion précédant cette bataille, aucune opinion n'était jugée supérieure, point particulièrement prisé puisque les participants ont 
eu l'impression de pouvoir débattre d'un sujet délicat sans être jugé. Ainsi, la discussion peut avoir lieu en accord ou en désaccord, l'importance étant de communiquer.

Nous examinons à présent une seconde scène significative. Il s'agit de la scène de l'intervention autochtone, toute dernière intervention de Trois. Les expressions choisies par les spectateurs ne font aucun doute sur ses effets. L'utilisation d'adjectifs qualificatifs laudatifs et de superlatifs tels que «magnifique », «très fort » ou encore «le plus intéressant » en atteste. Il est probable que cette intervention soit restée en mémoire puisqu'il s'agit de la scène finale. Néanmoins, nous avons remarqué que les spectateurs non-immigrants n'ont pas qualifié cette scène de marquante. Nous ne pouvons affirmer avec certitude la raison de cette différence entre immigrants et non-immigrants. Toutefois, ce qui est certain, c'est que les spectateurs immigrants ont reconnu dans le discours de l'autochtone leur propre quête identitaire et leur sentiment de déracinement, et ce, de façon beaucoup plus marquée que les non-immigrants. Le théoricien Pierre L'Hérault pense qu'une partie de la réponse au fait que les non-immigrants n'aient pas retenu cette scène est que la question autochtone se décline d'une manière particulière au Québec. Il croit que la relation trouble entre Québécois et Amérindiens résulte de différents historiques difficilement surmontables :

Le rapport à l'autre est beaucoup plus complexe quand il s'agit de la représentation amérindienne. D'abord, parce que la distinction entre les espaces y devient floue, voire inexistante, l'Amérindien et le Québécois habitant un même territoire, selon cependant des modes différents d'occupation. Et puis il y a cette histoire objectivement commune qui les unit dans un vaste malentendu! Cela suffit à suggérer l'inextricable nœud d'altérité et de même que forme ce lien projeté dans la figure de la gémellité. (163)

En outre nous remarquons qu'il existe en quelque sorte une compétition pour obtenir le titre de «minorité » entre les Québécois et les Amérindiens. De fait, à l'échelle du Canada, les Québécois sont perçus comme faisant partie d'une minorité en raison notamment de leur langue; les Amérindiens quant à eux sont une minorité et appartiennent aux Premières Nations. À l'échelle du Québec maintenant, les Québécois sont majoritaires, tandis que les Amérindiens restent minoritaires. L'immigrant, lui, peu importe l'endroit où il se trouve, reste toujours l'étranger, celui qui n'est pas d'ici, effet souvent renforcé par les propos de l'autre. Il est alors probable qu'il soit plus porté à se reconnaître dans le discours nomade et «minoritaire » de l'autochtone que dans le discours d'un non-immigrant. Parallèlement, le non-immigrant se 
reconnaîtrait moins dans le discours autochtone parce qu'il ne se trouve pas dans cette situation minoritaire à l'échelle de son territoire.

En ce qui concerne les scènes ayant marqué les spectateurs non-immigrants, notons une scène à la gestuelle fort symbolique, soit celle du décollage en avion. Voici comment Soleymanlou la décrit : «Je recule sur ma chaise en levant les bras vers le ciel, tout doucement, au ralenti. [...] Pendant une seconde ou deux, je suis comme en suspension dans l'avion !» (24). Annonçant «tout le reste de la pièce » selon Lucie, ce geste fait ressortir l'idée de départ qui est caractéristique de la perception que nous avons des immigrants. Pour celui qui n'a jamais immigré, l'image du départ reste très forte puisqu'il s'imagine l'immigrant devant quitter son pays et laisser tout derrière lui. Le non-immigrant retient souvent de cette expérience le côté traumatique. L'image de l'avion marque les esprits puisqu'elle symbolise justement ce départ, cette zone liminale en suspension entre le passé et le futur. Ici, ce n’est pas nécessairement le passage où Soleymanlou parle directement du moment où il embarquait dans l'avion pour retourner en Iran qui est retenu par les spectateurs, mais plutôt le geste qui y est associé. Jocelyn Lupien explique que les émotions sont un moyen privilégié pour partager les connaissances et que le langage verbal ne devrait pas être considéré comme l'unique méthode pour transmettre des messages. (239) L'émotion ressentie devant le geste de l'avion montre donc à quel point ce symbole peut avoir une grande portée. La scène de la bataille, mentionnée précédemment repose également sur ce principe. Michaël, un autre participant a également apprécié l'aspect non verbal de Soleymanlou. Notre entretien qui s'est déroulé deux semaines après la représentation indique que ce dont Michaël se souvenait le plus, correspondait aux gestes de Soleymanlou, comme si ses souvenirs reposaient sur des gestes et non des discours.

Nous avons par ailleurs été surprise de constater que beaucoup de participants ont mentionné l'importance de la mise en scène ou, du moins, ont reconnu des éléments qui lui étaient propres. Avec son décor constitué de plusieurs rangées de chaises, Soleymanlou voulait que, tour à tour, ce mobilier « représente, selon les scènes, une salle de classe, un aéroport, une salle d'attente, tous ceux et celles qui ne sont pas là, ceux et celles qui peuvent être assis là, à [s]es côtés » (11). Laure a apprécié cette idée puisqu'elle semble toute simple, mais peut amener plusieurs possibilités, ce qui en fait une «très bonne idée de départ ». Suzanne a aussi noté que la mise en scène de Soleymanlou favorisait la disparition du quatrième mur théâtral et les sujets avaient réellement l'impression que celui-ci s'adressait à eux : « le gars est là devant nous autres 
et il nous parle. On le voit respirer, il est là, il parle vraiment avec nous autres. On avait vraiment le feeling qu'il nous parlait ». D'ailleurs, à certains moments, Soleymanlou s'adresse réellement aux spectateurs en les incitant à la discussion. Le caractère performatif de la pièce peut avoir permis aux spectateurs de se rapprocher des artistes présents, notamment car la plupart du temps, les acteurs énoncent leurs répliques en regardant le public. Cet effet est particulièrement visible dans la première partie de la pièce où Soleymanlou est seul sur scène.

Dans un autre ordre d'idées, nous notons que les participants immigrants se sont beaucoup reconnus dans le discours de Soleymanlou, en particulier par rapport à la question d'être étranger dans un pays que considéré comme le sien. Citons dans un premier temps Jorge, qui résume très bien le sentiment général des immigrants : «Je me décris dernièrement comme un étranger éternel, ou constant, ou de laine ». D'autres participants ont fait part de cette impression sous une forme ou une autre, comme Julie qui indique que « Parfois, on oublie qu'on vient d'ailleurs parce que c'est ça notre ville, c'est notre pays, on y vit, c'est là où nos enfants grandissent, puis c'est toujours l'autre qui va te rappeler que tu es étrangère ». Nous avons remarqué que bien que le spectateur habite au Québec depuis plusieurs années, il demeure étranger et se le fait rappeler constamment.

Il est probable que le côté réaliste de la pièce joue un rôle significatif par rapport au phénomène d'identification aux personnages ressenti par les spectateurs. En fait, la plupart des immigrants interrogés ont mentionné se reconnaître d'une façon ou d'une autre dans les questionnements de Soleymanlou ou encore dans les situations vécues par les personnages immigrants dans la pièce. Il n'en reste pas moins que le réalisme de la pièce ne procède pas nécessairement d'une représentation vraisemblable des immigrants. Nous observons, à partir des commentaires de nos participants, que la vraisemblance des situations vécues par les acteurs immigrants dans la trilogie propose un discours double et nuancé. D’une part, ils trouvent que la représentation est très réelle et précise, mais aussi exagérée et peu représentative. Le commentaire de Jorge éclaire particulièrement cette situation : «on voyait ce qui était vrai ou on pouvait entrevoir ce qui était vrai ».

De leur côté, les sujets non-immigrants ont trouvé que la façon dont les immigrants étaient représentés dans la pièce pouvait parfois être comparée à ce qu'ils vivent dans leur vraie vie et non simplement sur scène. Pour Michaël, certaines situations sont représentatives, alors que d'autres le sont moins : «il y a des moments où ils font de l'humour et à d'autres moments 
où il fait une réelle réflexion [...] c'est assez représentatif si on ne prend pas les bouts où, justement, il [Mani] est dans l'exagération ». Suzanne, quant à elle, pense que le propos a été quelque peu grossi, mais elle apporte une nuance en disant que les acteurs « le vivent vraiment, mais que ça [les questions liées à l'identité] n'occupe pas nécessairement $100 \%$ de leur temps. Là, ils mettaient ça en premier plan comme si c'était le centre de leur vie, mais jusqu'à quel point c'est le centre de leur vie ? [...] Le but de la pièce était de nous parler de ça, donc c'est sûr que ça a été condensé ». Nous nous attendions déjà à ce type de réponse de la part des nonimmigrants. Il est possible que, n'ayant pas eux-mêmes fait l'expérience de l'immigration, les Québécois ne puissent pas appréhender le bagage émotionnel de cette expérience. Il se peut aussi que les sujets non-immigrants se rendent compte qu'il s'agit d'une pièce de théâtre dans laquelle chacun a dû faire des concessions. Nous ne croyions toutefois pas constater que les spectateurs immigrants partageaient cette lucidité devant la pièce de théâtre. Cela peut s'expliquer grâce au commentaire de Soleymanlou qui précise à plusieurs endroits dans Trois qu'il s'agit de son histoire et de son expérience personnelle.

Pourrions-nous alors dire avec certitude que la trilogie est représentative de ce que les immigrants vivent réellement? La réponse est ici plus nuancée que ce que nous aurions pu croire. Les sujets immigrants autant que les non-immigrants apportent une réponse nuancée à cette question et mettent en avant le fait qu'il est impossible que le théâtre soit une copie conforme de la réalité. En fait, le théâtre, par définition, sert souvent de miroir à la société et « est chargé de la responsabilité de refléter le vécu humain dans toute sa complexité. [...] Une telle orientation vise [alors] à sortir de l'illusion pour s'inscrire dans le réel ou, du moins, pour simuler le réel ». (Dopinescu 45-46) Les termes « refléter» et «simuler» présentent bien de quelle façon le théâtre ne fait qu'imiter une certaine réalité humaine, cette dernière étant si complexe qu'on ne peut en saisir la totalité sur scène. Par ailleurs,

Il est important de se rappeler que le théâtre est une construction d'images (nouvelles) à partir des images du réel; la représentation est donc un univers de présences qui font sens. Autrement dit, dans le thêâtre traditionnel, les figures scéniques dépassent leur qualité d'être-là et, suivant le projet de la mise en scène, elles aspirent au statut de représentations, d'univers possibles, de "vérités » auxquelles on croit, auxquelles on tâche de s'identifier et sur lesquelles on projette des désirs. (47)

C'est ce dont les spectateurs ont été témoins ; ils ont vu des représentations plus ou moins fidèles de la réalité, mais ils veulent croire à ces «vérités », s'identifier à elles et espérer que cela 
contribue à réfléchir à la question de l'immigration. Il ne semble donc pas nécessaire que tout soit en tous points fidèle à la réalité pour que le spectateur adhère aux propos véhiculés dans le spectacle. L'important est d'y croire et de comprendre que ces images sont tirées du réel. Même si la pièce résulte la plupart du temps d'une expérience personnelle de l'auteur ou des acteurs, et bien que les spectateurs interrogés ne se soient pas identifiés d'emblée à un certain personnage — outre certains sujets immigrants, comme Jorge qui s'est reconnu dans Mani —, ils ont réussi à apprécier la pièce et à en tirer des réflexions personnelles. Ce phénomène s'explique notamment parce que :

Cette intimité entre le vécu des comédiens et celui des spectateurs assure la toute première identification du public à une prise de parole qui pourrait tout aussi bien venir «de cousins qui vivent la même affaire qu'eux autres. Le «nous» de l'organisation se fusionne alors au «nous » de la communauté. Ce positionnement entre la culture organisationnelle et la culture du milieu prédispose déjà à la représentativité de la création, indépendamment de toute technique discursive : vraisemblance, traitement « réaliste », etc. (Parent 200)

Comme Trois met en jeu beaucoup de références tirées de la réalité québécoise, le spectateur est alors susceptible d'effectuer des comparaisons entre ce qu'il voit et ce qui est présent dans la société. Cette simulation de la réalité dans la pièce joue-t-elle un rôle dans la perception de l'échange intraculturel?

Cela nous amène à la dernière question que nous souhaitons aborder qui est de savoir si une pièce comme Trois peut, selon les spectateurs, avoir une réelle influence sur le public et indiquer que l'échange intraculturel est réussi. Comme Trois s'inscrit dans le processus intraculturel par lequel la rencontre entre des cultures différentes au sein d'une nation peut être facilitée, il nous semblait intéressant de vérifier si le public la voyait ainsi. En fait, les transferts culturels qui résultent de pièces telles que celle de Soleymanlou :

[...] sont le lieu d'exploration et d'interrogation de l'altérité, des processus identitaires dans et par lesquels se construisent, se déconstruisent et se reconstruisent, les représentations de soi et de l'autre. Elles permettent aussi d'analyser le jeu des identités, individuelles et collectives, imprégnées par les différences linguistiques, régionales, culturelles, politiques, sociales et nationales. Tout s'y retrouve confondu, mais aussi distinct, les conflits, les pouvoirs de domination, les confrontations, les douleurs de l'exil, de la souffrance, de la rupture, le déracinement et l'enracinement. [Elles] se présentent aussi comme des sources ou des occasions de restructuration des identités en contact, par divers modes, d'appropriation, d'acculturation, de métissage. (Moisan 72) 
La majorité des participants interrogés pense que ce type de spectacle peut avoir une influence positive sur la société québécoise parce qu'il permet de mieux comprendre ce que vivent les immigrants, même si ce n'est pas identique en tous points à leur réalité. Ils s'appuient principalement sur ce que la pièce a eu comme effet sur eux pour appuyer leurs dires. Par exemple, Suzanne explique que Trois lui a fait «ouvrir les horizons » en l'amenant à découvrir une réalité qu'elle ne soupçonnait pas. Depuis qu'elle a vu la pièce, elle se sent plus «empathique» envers les gens issus de l'immigration. De son côté, Louise «a changé beaucoup » la vision qu'elle avait des jeunes immigrants et a réalisé qu'elle avait peut-être des jugements, des préjugés envers certains groupes.

Par ailleurs, Lucie précise aussi que Trois a eu un impact «non seulement [...] entre les gens de différentes cultures d'abord sur la scène, mais ça crée ça [un impact] aussi avec les spectateurs, c'est clair. » Notons ici qu'elle mentionne ce qui se passe sur scène et entre les spectateurs. En fait, elle perçoit la dualité du discours intraculturel : le rapport se fait entre les acteurs puis avec les spectateurs. Pour le théoricien Marco de Marinis :

Cette réciprocité des regards est encore plus forte parce qu'on peut parler d'un double désir de l'Autre, ou de l'altérité [...] Ce qui rapproche les maîtres de la scène contemporaine, c'est l'effort de penser et de réaliser un théâtre comme une rencontre de connaissance, comme une expérience interhumaine authentique, une découverte et une transformation de soi, rendue possible par le fait qu'elle n'est pas reconnaissance de l'identique et du déjà connu, mais confrontation et exploration de l'altérité, donc de l'inconnu et même de l'obscur, et cela, soit pour l'acteur, soit pour le spectateur. (88)

En rapprochant des acteurs issus de divers milieux culturels et en les présentant tous sur une même scène, le spectateur peut alors voir en quoi consiste cet échange intraculturel. D'autre part, le rapport intraculturel s'effectue dans la conception même de la pièce : une telle pièce, selon Lucie, «s'est faite ensemble, ça a pris du temps. Ça veut dire que ce que ça montre, c'est qu'on est peut-être, dans certains moments, rendu pas mal plus loin que ce que d'autres événements pourraient nous faire croire. » Le mot-clé se trouve justement ici dans cet «ensemble », cet aspect de réalisation collective qui amène à créer « une pièce qui nous amène à réfléchir où on est rendu comme société ».

Devant la cohabitation scénique d'acteurs issus de diverses communautés culturelles, le spectateur se trouve confronté à la diversité présente dans cette nation et assiste plus précisément à l'ouverture d'une discussion portant tant sur les origines des acteurs que sur les défis que 
certains ont vécus en raison de leurs traits physiques ou de leur arrivée dans un nouveau pays. Par moment, il semble que «c'était peut-être un peu trop » (Suzanne) en raison de la cacophonie lorsque les acteurs parlent les uns par-dessus les autres, mais en d'autres occasions une voix s'élève et explique son cheminement personnel. Ainsi, le spectateur est amené à entrevoir les pensées de cette personne. À travers cela se dessine une rencontre intraculturelle où les communautés culturelles cohabitent sur une même scène, dans un même espace public. Bien que le consensus ne soit pas toujours atteint lorsque vient le temps de discuter de certains sujets difficiles — il suffit de penser à la scène à propos des accommodements raisonnables qui se termine par une épique bataille — , ceux qui veulent prendre la parole peuvent le faire sans égard à l'opinion qu'ils expriment. L'image devant soi est celle d'une société plurielle où chacun jeune ou âgé, immigrant ou non-immigrant — a son propre vécu et où pourtant le dialogue peut avoir lieu. C'est dans ce contexte que se produirait un dialogue intraculturel : entre les acteurs, les nations diverses se rencontrent et échangent sur ce qui les relie ou les divise, mais le tout dans un contexte d'égalité. Immigrants et non-immigrants se côtoient, jouent ensemble, discutent ensemble, vivent ensemble. L'accent est donc mis sur un dialogue s'effectuant à grande échelle et non simplement entre de petits groupes d'individus. Le spectateur est alors susceptible d'effectuer des comparaisons entre ce qu'il voit et ce qui est présent dans la société actuelle ; il pourra par après reproduire ce à quoi il a assisté dans ses rapports avec l'Autre et pourra faire preuve d'une plus grande ouverture d'esprit. Ainsi, les «barrières » entre les communautés s'amincissent, car nous apprenons à mieux connaître l'Autre.

Certains participants ont évoqué cette «connaissance» de l'Autre. Pour eux, l'échange intraculturel est réussi parce que la trilogie permettait de mieux « connaître » ou «comprendre » l'Autre. L'échange intraculturel se produit plus dans une visée pédagogique que discursive : à la suite de la représentation, le spectateur saisit ce que l'Autre vit et comprend mieux sa réalité. Mais il n'est pas nécessaire que cela s'accompagne d'une remise en question des valeurs ou d'une nouvelle ouverture à l'Autre. En ce sens, peu de participants ont réellement noté chez eux un changement dans leur comportement ou leur façon de voir les immigrants : ils ont surtout appris ce en quoi consistaient l'expérience migratoire et l'adaptation dans la société d'accueil que devient le Québec. Certes, le changement n'est pas immense, mais, d'après Marios Koukounaras-Liagis, «ces changements, aussi petits soient-ils, sont d'une importance capitale 
pour les sociétés étant donné que les grands changements au niveau social peuvent être le résultat de changements mineurs à un niveau personnel » (86).

En bref, des pièces comme Trois favorisent un climat d'échange et de partage entre différentes communautés culturelles. Elles sont donc nécessaires à toute société qui essaie de mettre en place un dialogue intraculturel dans lequel chacun, peu importe son origine, détient une place équivalente et où l'accent est mis sur un partage des valeurs communes. Quand le spectateur assiste à ce type de spectacle, il est amené à réfléchir à ce qu'il a vu, à ses rapports à l'Autre et même à son identité. S'il poursuit ses réflexions, alors il peut réellement remarquer un changement dans ses valeurs et faire preuve d'une plus grande ouverture d'esprit. En tant que société, l'ouverture d'esprit est une des qualités les plus importantes. Ouvrons donc la porte à la diversité, et passons par le théâtre pour y arriver. Car il n'y a rien de mieux que d'être confronté à l'Autre dans un tel cadre pour comprendre à quel point il nous ressemble. Et quand il n'y aura plus de différences entre l'Autre et nous, nous pourrons finalement dire que nous sommes dans un pays égalitaire où marge et norme ne font qu'un.

\section{Bibliographie}

Bharucha, Rustom. Theatre and the World : Performance and Politics of Culture. Londres : Manohar, 2005.

De Marinis, Marco. «L'expérience de l'altérité. Le théâtre entre interculturalisme et transculturalisme». L'Annuaire théâtral: revue québécoise d'études théâtrales 26 (1999) : 84-102.

Dospinescu, Liviu. «Effet de présence et non-représentation dans le théâtre contemporain ». Tangence. 88 (2008) : 45-61.

Knowles, Ric. Theatre and Interculturalism. New York : Palgrave Macmillan, 2010.

Koukounaras-Liagis, Marios. «Can an Educational Intervention, Specifically Theatre in Education, Influence Student's Perceptions of Attitudes to Cultural and Religious Diversity? A Socio-educational Research ». British Journal of Religious Education 33-1 (2011) : 75-89.

L'Hérault, Pierre. «L'espace immigrant et l'espace amérindien dans le théâtre québécois ». Nouveaux regards sur le théâtre québécois. Dir. Betty Bednarski et Irene Oore, Montréal : XYZ, coll. « Documents », 1997. 
Lupien, Jocelyn. «Le déverrouillage sensoriel et identitaire». Dir. Pierre Ouellet. Le soi et l'autre. L'énonciation de l'identité dans les contextes interculturels. Québec: PU Laval, coll. «Intercultures », 2002.

Moisan, Clément. Écritures migrantes et identités culturelles. Québec: Nota Bene, coll. «Études », 2008.

Soleymanlou, Mani. Trois. Montréal : L'instant même, coll. «L'instant scène », 2014. 